\title{
ADOLESCÊNCIA, TRANSPLANTE HEPÁTICO E ADESÃO À MEDICAÇÃO
}

\author{
Adolescence, liver transplantation and medication adherence
}

\begin{abstract}
Beatriz Elizabeth Bagatin Veleda Bermudez¹, Maria de Fátima Joaquim Minetto², Mônica Beatriz Parolin ${ }^{3}$, Júlio Cezar Uilli Coelho ${ }^{4}$, Mariane Bagatin Bermudez ${ }^{5}$, Adriane Celli ${ }^{6}$
\end{abstract}

\section{RESUMO}

As transformações biopsicossociais da adolescência colocam os adolescentes transplantados de fígado em maior risco de não aderência ao tratamento e piora da evolução, quando comparados a outros grupos etários. Objetivo: Verificar a influência de fatores psicológicos do paciente e sua família na aderência ao imunossupressor após o transplante hepático. Métodos: Sete instrumentos psicológicos foram aplicados a 30 pacientes transplantados hepáticos adolescentes e adultos jovens (12 a 30 anos): escala de autoestima, escala de resiliência, escala de qualidade da interação familiar, escala de satisfação com a vida, escala de orientação de vida, escala de autoeficácia percebida, escala de exigência e responsividade e escala de qualidade de interação familiar. Resultados: Na avaliação por regressão logística multivariada, as variáveis orientação de vida e resiliência foram as principais características que interferiram na rejeição clínica ( $p=0,03$ ). As variáveis do pai que atuam na boa aderência $(\mathrm{p}=0,03)$ foram: comunicação negativa, responsividade, clima conjugal positivo, punição física e comunicação positiva, enquanto que envolvimento, sentimento dos filhos e comunicação positiva aumentaram a rejeição clínica ( $p$ $<0,01)$. Para a mãe, responsividade, comunicação positiva e punição física tended to adhesion ( $\mathrm{p}=0,06)$, enquanto que a punição física e a comunicação negativa aumentaram a possibilidade de rejeição clínica $(p=0,02)$. Conclusões: O perfil psicológico do paciente e da família interfere na adesão ao tratamento imunossupressor e na rejeição pós-transplante hepático e poderia ser utilizado para triagem de pacientes com risco de má aderência ao tratamento imunossupressor e consequente rejeição ao enxerto.

Descritores: Adolescência; Transplante de Fígado; Adesão à Medicação; Adaptação Psicológica Relações Familiares; Rejeição de Enxerto.

\section{Instituições:}

${ }^{1}$ Saúde da Criança e do Adolescente, Hospital de Clínicas, Universidade Federal do Paraná (UFPR), Curitiba, PR

${ }^{2}$ Departamento de Educação, Universidade Federal do Paraná (UFPR), Curitiba, PR

${ }^{3}$ Programa de Transplante Hepático, Hospital de Clínicas, Universidade Federal do Paraná (UFPR), Curitiba, PR

${ }^{4}$ Departamento de Cirurgia do Aparelho Digestivo e Transplante Hepático, Hospital de Clínicas, Universidade Federal do Paraná (UFPR), Curitiba, PR

${ }^{5}$ Acadêmica de Medicina da Universidade da Região de Joinville - Univille, Joinville, SC

${ }^{6}$ Disciplina de Gastro e Hepatopediatria, Departamento de Pediatria,

Hospital de Clínicas, Universidade Federal do Paraná (UFPR), Curitiba, PR

\section{Correspondência:}

Beatriz Elizabeth Bagatin Veleda Bermudez

Rua Desembargador Westphalen, 15 - Cj.1405, CEP 80010-110 - Curitiba/PR

Fone: (41) 3224-6476

E-mail: beatriz_bvb@hotmail.com

Recebido em: 13/12/2011

Aceito em: 19/01/2012

\section{INTRODUÇÃO}

O transplante hepático é o tratamento de eleição nas doenças avançadas do fígado, alcançando índices de sobrevida em um ano de 83 a 91\% e, em cinco anos, de 82 a 84\%, dependendo da idade do receptor. ${ }^{1}$ Entre os desafios dos centros de transplante hepático estão o manejo e a melhora na transferência dos adolescentes aos serviços dos adultos. ${ }^{2}$

A adolescência é uma etapa crucial e bem definida do processo de crescimento e desenvolvimento, cuja marca registrada é a transformação ligada aos aspectos físicos e psíquicos do ser humano, com intenso desenvolvimento cerebral até a terceira década de vida. ${ }^{3-5}$ Esse período é extremamente relevante para a construção do sujeito individual e social, devendo, porém, ser considerados a vulnerabilidade e o risco do uso indevido de drogas, doenças sexualmente transmissíveis, AIDS e gravidez não planejada, além de apresentar pico de incidência para doença mental.

Paralelamente às modificações corporais, como crescimento físico, eclosão hormonal, maturação sexual (puberdade), evoluem as modificações psicossociais, ${ }^{6}$ no que convencionaram chamar de síndrome da adolescência normal, que são: busca da identidade, tendência grupal, desenvolvimento do pensamento abstrato, vivência temporal singular, variações do humor, evolução da sexualidade, separação progressiva dos pais, crises religiosas, atitude social reivindicatória e manifestações contraditórias de conduta. 
A necessidade de manter um compromisso com um acompanhamento médico contínuo é um fator adicional para o estresse próprio dessa fase da vida.?

Adolescentes receptores de transplante de órgão sólido, com idade entre 18 e 24 anos, apresentam o dobro da taxa de mortalidade em relação àqueles entre 12 e 17 anos e quatro vezes maior do que os de 10 a 14 anos. ${ }^{8} \mathrm{O}$ uso de drogas também aumenta e a taxa de suicídio é três vezes maior que a de adolescentes entre 12 e 17 anos ${ }^{5}$ e mais de um terço dos adolescentes não aderem ao tratamento. ${ }^{8} \mathrm{~A}$ tendência normal ao questionamento e desafio à autoridade podem predispor esses pacientes a aceitar com desdém as orientações médicas e o tratamento. ${ }^{8}$

Embora em alguns trabalhos, adolescentes com doenças crônicas pareçam apresentar maior risco, ${ }^{9}$ um estudo com 116 pacientes com idade $21+/-4$ anos observou que o comportamento de risco não apresentou diferença em relação a seus pares normais referente ao uso de tabaco (11\%) e maconha (11\%), mas encontrou taxas significativamente menores nesses pacientes quanto ao uso de álcool (43\% nunca usaram e $50 \%$ bebem eventualmente em ocasiões especiais). ${ }^{10}$

\section{PACIENTES E MÉTODOS}

$\mathrm{O}$ estudo contou com 30 pacientes transplantados de fígado que, no momento do estudo, estavam com idade entre 12 e 30 anos. $\mathrm{O}$ transplante ocorreu na infância em sete pacientes, na adolescência inicial em nove pacientes, na adolescência média em oito pacientes e, na adolescência final, em seis pacientes. $\mathrm{O}$ imunossupressor mais usado foi o tacrolimus. Quanto ao tempo decorrido do transplante: nove pacientes tinham até cinco anos, nove tinham entre cinco e dez anos e doze pacientes estavam transplantados há mais de dez anos. $\mathrm{O}$ seguimento foi no ambulatório de transplante hepático pediátrico e até mesmo adulto, devido ao hospital limitar a idade da pediatria em 14 anos, diferente da Organização Mundial de Saúde e do Ministério da Saúde do Brasil, que estipula o atendimento pediátrico até 19 anos, 11 meses e 29 dias. Apesar disso, muitos pacientes permaneciam no ambulatório pediátrico além dos 14 anos, por solicitação própria ou dos pais, por não se apresentarem clínica e/ou laboratorialmente estáveis, ou mesmo pela equipe não perceber maturidade para transição ao ambulatório dos adultos. $\mathrm{O}$ limite de idade foi 30 anos.

A doença que motivou o transplante foi a hepatite autoimune, em nove pacientes; colangite esclerosante, em cinco; cirrose criptogenética, em cinco; deficiência de alfa-1-antitripsina, em quatro; hepatite B em dois; hepatite fulminante em dois; doença de Wilson, em um e, em outro, doença de Alagille.

Os pacientes e responsáveis foram convidados a participar da pesquisa e aqueles que concordaram e tinham boa escolaridade (oito anos ou mais de educação formal) leram e responderam os questionários, enquanto alguns com baixa escolaridade (menos de oito anos de educação formal) necessitaram de ajuda, como a leitura das questões.

Foram utilizados sete instrumentos para avaliação do perfil psicológico de 30 pacientes adolescentes e adultos jovens (12-30 anos): escala de satisfação com a vida, ${ }^{11}$ teste de orientação da vida (que avalia o otimismo do paciente em relação a eventos futuros), ${ }^{12}$ escala de resiliência, ${ }^{13,14}$ escala de autoestima, ${ }^{15,16}$ escala de autoeficácia geral percebida (que avalia autoconfiança, adaptação e enfrentamento), ${ }^{17}$ escala de exigência e responsividade (avaliaram o quanto o paciente percebia seus pais quanto às normas, supervisão e afeto) ${ }^{18,19}$ e escala de qualidade na interação familiar - EQIF. ${ }^{20,21}$ Essa última continha 40 questões divididas em nove dimensões. Seis delas abordavam os aspectos "positivos": envolvimento, regras e monitoria, modelo moral, clima conjugal positivo, comunicação positiva dos filhos e sentimentos do filho. As outras três referiam- se aos aspectos "negativos": clima conjugal negativo, comunicação negativa dos pais e punição física. ${ }^{20,21}$

Os dados foram analisados pelo programa Statistical Program for Social Sciences 13 (SPSS 13) e programa Statistica.

\section{RESULTADOS}

A figura 1 mostra a evolução dos 30 pacientes. A aderência ao tratamento imunossupressor foi de $70 \%$. O medicamento imunossupressor mais usado pelo grupo foi o tacrolimus em todos os pacientes, exceto um, que foi retransplantado após um ano, devido à rejeição, e usa sicrolimus.

Uma paciente com 23 anos desenvolveu hepatite aguda fulminante após seis anos de transplante de medula óssea, devido à leucemia refratária, com boa evolução.

Figura 1 - Aderência, rejeição clínica e rejeição na biópsia dos 30 pacientes da amostra

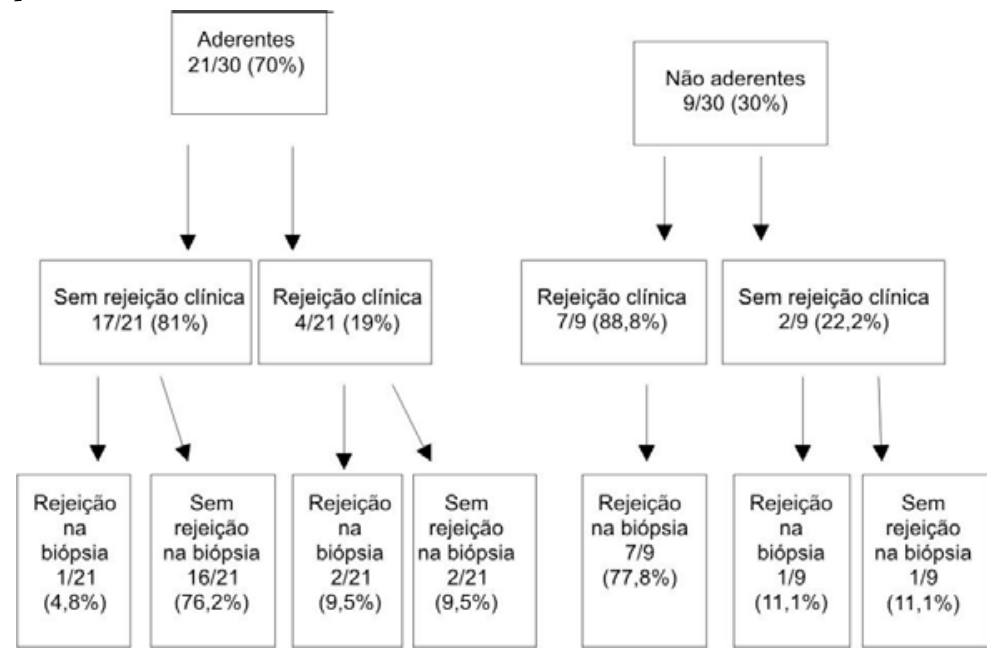

Com relação à análise isolada das variáveis do perfil psicológico dos pacientes, não houve diferença significativa quanto à satisfação com a vida, orientação de vida, resiliência, autoestima e autoeficácia no teste de Mann-Whitney, com a presença ou ausência de aderência, rejeição clínica (exames laboratoriais mostrando disfunção hepática) ou rejeição à biópsia, como se pode observar nas tabelas 1,2 e 3 . Na análise das variáveis da família, a punição física praticada pela mãe aumentou a rejeição clínica $(\mathrm{p}=0,03)$. Os pacientes analisados tiveram ao menos uma repetição de rejeição clínica anotada no prontuário do serviço de transplante hepático, quando foi adaptada a dose do imunossupressor, consultas com intervalos mais próximos com checagem de novos exames, avaliação de situações clínicas prejudicando absorção e meia vida da imunossupressão e programação da biópsia hepática.

$\mathrm{Na}$ regressão logística multivariada, orientação e resiliência foram as principais variáveis que interferiram na rejeição clínica $(\mathrm{p}=0,03)$ e foi observada uma correlação positiva da aderência $(p=0,03)$ com as variáveis do pai de comunicação positiva $(\mathrm{OR}=0,5[0,2-0,9])$ e negativa $(\mathrm{OR}=0,5[0,3-0,9])$, responsividade $(\mathrm{OR}=1,4[0,9-2,0])$ e clima positivo $(\mathrm{OR}=0,4[0,2-0,9])$ e com as variáveis da mãe $(\mathrm{p}=0,06)$ de exigência $(\mathrm{OR}=1,5[0,9-2,4])$, punição física $(\mathrm{OR}=1,7$ $[0,5-1,2])$ e comunicação positiva $(\mathrm{OR}=1,2[0,9-1,4])$, mostradas na figura 2. As variáveis da mãe $(p=0,02)$, que aumentaram a rejeição clínica, foram: punição física $(\mathrm{OR}=1,5[0,9$ a 2,3]) e comunicação negativa $(\mathrm{OR}=1,3[0,9$ a 1,7$])$; e as variáveis do pai $(\mathrm{p}<0,01)$ foram: envolvimento $(\mathrm{OR}=1,6[0,3-0,8])$, sentimento dos filhos $(\mathrm{OR}=1,6$ $[1,0-2,5])$ e comunicação positiva $(\mathrm{OR}=2,7[1,2-5,8])$, mostradas na figura 3 . 
Tabela 1 - Comparação dos escores das características do perfil psicológico dos pacientes entre os grupos com e sem rejeição clínica

\begin{tabular}{|c|c|c|c|c|c|c|}
\hline Aderência & Média & Mínimo & Máximo & $\begin{array}{l}\text { Desvio } \\
\text { Padrão }\end{array}$ & $\mathrm{U}$ & $\mathbf{P}$ \\
\hline \multicolumn{7}{|l|}{$\begin{array}{l}\text { Satiasfação } \\
\text { com a Vida }\end{array}$} \\
\hline SIM & 26,1 & 17 & 31 & 5,4 & 84 & 0,65 \\
\hline NÃO & 26,5 & 14 & 35 & 5,1 & & \\
\hline \multicolumn{7}{|l|}{$\begin{array}{l}\text { Orientação } \\
\text { da Vida }\end{array}$} \\
\hline SIM & 18 & 9 & 24 & 4,5 & 67 & 0,22 \\
\hline NÃO & 16 & 12 & 21 & 3,0 & & \\
\hline \multicolumn{7}{|l|}{ Resiliência } \\
\hline SIM & 138 & 110 & 175 & 16,5 & 91,5 & 0,89 \\
\hline NÃO & 139 & 128 & 157 & 9,8 & & \\
\hline \multicolumn{7}{|l|}{ Autoestima } \\
\hline SIM & 35,8 & 29 & 43 & 5,0 & 71 & 0,30 \\
\hline NÃO & 38,6 & 29 & 43 & 5,0 & & \\
\hline \multicolumn{7}{|l|}{ Autoeficácia } \\
\hline SIM & 33,0 & 23 & 40 & 4,5 & 93,5 & 0,96 \\
\hline NÃO & 32,3 & 18 & 40 & 6,8 & & \\
\hline
\end{tabular}

NOTA: Teste de Mann-Whitney

Tabela 2 - Comparação dos escores das características do perfil psicológico dos pacientes entre os grupos aderentes e não aderentes

\begin{tabular}{|c|c|c|c|c|c|c|}
\hline Aderência & Média & Mínimo & Máximo & $\begin{array}{l}\text { Desvio } \\
\text { Padrão }\end{array}$ & U & $\mathbf{P}$ \\
\hline \multicolumn{7}{|l|}{$\begin{array}{l}\text { Satiasfação } \\
\text { com a Vida }\end{array}$} \\
\hline SIM & 25,8 & 14 & 32 & 4,8 & 85 & 0,42 \\
\hline NÃO & 26,9 & 17 & 35 & 5,8 & & \\
\hline \multicolumn{7}{|l|}{$\begin{array}{l}\text { Orientação } \\
\text { da Vida }\end{array}$} \\
\hline SIM & 18,2 & 9 & 24 & 4,8 & 70,5 & 0,14 \\
\hline NÃO & 15,9 & 12 & 21 & 2,5 & & \\
\hline \multicolumn{7}{|l|}{ Resiliência } \\
\hline SIM & 134,8 & 110 & 156 & 13,3 & 68 & 0,12 \\
\hline NÃO & 145 & 128 & 175 & 15,2 & & \\
\hline \multicolumn{7}{|l|}{ Autoestima } \\
\hline SIM & 37,8 & 29 & 50 & 6,8 & 102 & 0,93 \\
\hline NÃO & 37,6 & 30 & 47 & 5,6 & & \\
\hline \multicolumn{7}{|l|}{ Autoeficácia } \\
\hline SIM & 33,1 & 28 & 40 & 3,6 & 93,5 & 0,64 \\
\hline NÃO & 32,4 & 18 & 40 & 7,3 & & \\
\hline
\end{tabular}

NOTA: Teste de Mann-Whitney
Tabela 3 - Comparação dos escores das características do perfil psicológico entre os grupos com e sem rejeição na biópsia

\begin{tabular}{lcccccc}
\hline Aderência & Média & Mínimo & Máximo & $\begin{array}{c}\text { Desvio } \\
\text { Padrão }\end{array}$ & U & P \\
\hline $\begin{array}{l}\text { Satiasfação } \\
\text { com a Vida }\end{array}$ & & & & & & \\
SIM & 26,2 & 14 & 35 & 5,3 & 104 & 0,88 \\
NÃO & 26,2 & 17 & 31 & 5,0 & & \\
Orientação & & & & & & \\
da Vida & & & & & & \\
SIM & 17,4 & 9 & 24 & 4,8 & 106,5 & 0,95 \\
NÃO & 17,3 & 12 & 23 & 3,3 & & \\
Resiliência & & & & & & \\
SIM & 136,6 & 110 & 175 & 16,8 & 84 & 0,32 \\
NÃO & 141,5 & 128 & 157 & 10,7 & & \\
Autoestima & & & & & & \\
SIM & 37,7 & 29 & 50 & 7,1 & 106 & 0,95 \\
NÃO & 37,9 & 30 & 45 & 5,2 & & \\
Autoeficácia & & & & & & \\
SIM & 33,5 & 28 & 40 & 3,8 & 108 & 1,00 \\
NÃO & 31,9 & 18 & 40 & 6,7 & & \\
\hline NOTA: Teste & & & & & &
\end{tabular}

NOTA: Teste de Mann-Whitney

Figura 2 - Perfil da família e aderência ao imunossupressor

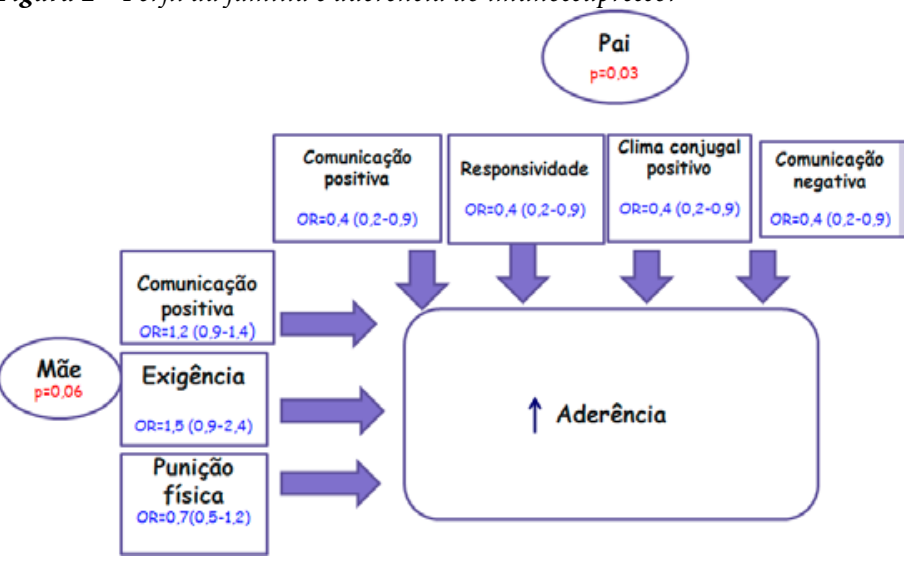

Figura 3 - Perfil da família e rejeição clínica

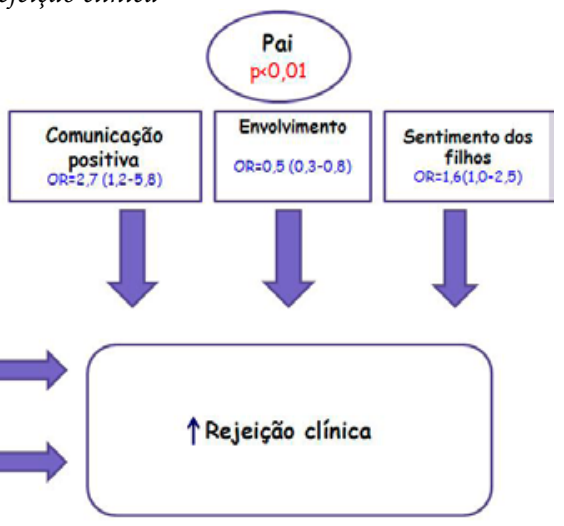




\section{DISCUSSÃO}

A aderência mostrou-se alta $(70 \%)$ na amostra de adolescentes e jovens transplantados de 12 a 30 anos, pois, considerando a faixa etária, a não aderência é maior na adolescência (38 a 64\%), menor nas crianças (17\%) e intermediária nos adultos (15 a $25 \%$ ), podendo variar de 7 a $70 \%{ }^{7,22,23}$

Os fatores de risco para não adesão ao tratamento citados pela Organização Mundial de Saúde são numerosos, envolvendo também fatores socioeconômicos, fatores relacionados ao paciente, à doença, ao serviço de saúde e ao tratamento; a não aderência é um processo dinâmico. $\mathrm{O}$ paciente pode estar aderente à medicação imunossupressora em um momento e não aderente em outro, pois não existe um método que meça continuamente o nível das drogas imunossupressoras. ${ }^{23}$

A comunicação médico-paciente na adolescência é uma estratégia imprescindível no tratamento de doenças crônicas com necessidade de bom vínculo entre a mãe e o filho enfermo e do paciente ser elevado à posição de comunicante precocemente para melhorar seu desenvolvimento psicológico e adesão, concluindo que a qualidade da comunicação determina a qualidade da adesão. ${ }^{24}$

Somente a punição física praticada pela mãe sobressaiu-se na análise isolada das variáveis, enquanto as demais necessitaram ser associadas por regressão logística.

Orientação de vida e resiliência interferiram na rejeição clínica. Uma orientação de vida otimista está relacionada com boa saúde física e mental, enquanto uma orientação pessimista relaciona-se com depressão, ansiedade e prática de comportamentos de risco. ${ }^{25}$

Não foram encontradas pesquisas publicadas sobre essas variáveis do perfil psicológico e a evolução no transplante hepático, porém um estudo sobre o otimismo (que pode ser avaliado pelo teste de orientação da vida), em 5634 adolescentes da população em geral, evidenciou que essa qualidade protege contra depressão, uso pesado de drogas e do comportamento antissocial, sugerindo incluir o otimismo nas intervenções na adolescência. ${ }^{26}$

Em relação à autoestima de 55 pacientes transplantados de fígado de 5 a 18 anos, quando comparados a 391 crianças e adolescentes da população em geral, seus escores físicos foram menores, porém a autoestima e a saúde mental demonstraram-se semelhantes nos dois grupos. Já os pais desses receptores de fígado apresentaram níveis mais altos de estresse e de descontinuidade de suas atividades do que os pais da população em geral. ${ }^{27}$

Não existem estratégias bem estabelecidas para boa adesão ao tratamento clínico e cuidados de saúde em doença crônica, pois, mesmo a não intervenção foi identificada como sendo bem estabelecida, porém estratégias que promovam organização ajudam a aderência na asma, estratégias comportamentais ajudam na evolução da artrite reumatoide juvenil e aprender a lidar com a medicação e alterações da glicemia auxiliam no diabetes mellitus tipo $1 .^{28}$

O desenvolvimento da autonomia é semelhante nos adolescentes saudáveis e com diabetes mellitus tipo 1 e a divisão de responsabilidade com os pais, com supervisão adequada, melhora a saúde mental dos filhos; autocuidado, assim como o envolvimento dos pais no manejo da doença, está associado com melhor controle metabólico, porém há comprometimento do controle do diabetes quando os adolescentes resistem a esses esforços dos pais, surgindo conflitos familiares. ${ }^{29}$

Os pais devem continuar a monitorar seus adolescentes e estabelecer regras para eles na adolescência, mesmo que a crença dos filhos de obedecê-los diminua no transcorrer dessa fase. ${ }^{30}$

Há uma conexão entre o estado de saúde física e mental observada em 24 pacientes com 18 e 22 anos transplantados de órgão sólido na infância: o poder preditivo de autoestima para explicar o afeto positivo (60\% da variância total) e o estado de saúde física, autoconceito e tempo na lista de espera explicaram o afeto negativo (71\% da variância). ${ }^{31}$

Dois fenômenos foram observados na entrevista com oito famílias com filhos submetidos a transplante hepático: a vida controlada pelo transplante (que representa a vulnerabilidade ao vivenciar incertezas e medo constante pela doença da criança) e a luta para resgatar a autonomia e enfrentar o estresse e sofrimento. ${ }^{32}$

A punição física e a comunicação negativa praticada pela mãe aumentam a rejeição clínica, apesar de mães de crianças com doenças crônicas usarem um número menor de práticas coercitivas, como punição física e privação/castigo quando comparadas com mães de filhos saudáveis. ${ }^{33}$

Mães usuárias de um serviço público de saúde usam como práticas educativas as estratégias indutivas, de distração, de evitação de conflitos e as de força coercitiva, motivadas por influências familiares, as experiências com o primeiro filho e a busca da melhor maneira de agir. ${ }^{34} \mathrm{~A}$ naturalização do uso de práticas de força coercitiva evidencia a ocorrência de violência física e psicológica contra a criança no contexto familiar, justificadas pelas mães como a necessidade de educar, porém com sentimento de culpa e mal-estar por terem batido nos filhos. ${ }^{34}$ As práticas que caracterizam a violência psicológica não foram admitidas como prejudiciais, o que demonstra a necessidade de maior divulgação de conhecimento científico e criação de espaços de discussão e reflexão sobre práticas educativas familiares nas comunidades, escolas, creches, unidades de saúde, hospitais, entre outros. ${ }^{34}$

O uso da punição física está associado a pontos negativos na interação pais-filhos e com colegas, propiciando comportamentos agressivos, baixo autoconceito e baixa autoestima, que está associada a problemas sociais contemporâneos, como abuso de drogas, gravidez precoce, fracasso escolar e delinquência. ${ }^{35}$

Há significativa melhora da evolução quando se associa ao tratamento medicamentoso e psicológico do filho um treinamento de habilidades parentais por técnicas comportamentais para disciplina, melhorando o apego e envolvimento para o desenvolvimento social e emocional em um estudo com 155 mães de crianças com déficit de atenção e hiperatividade. ${ }^{36}$

Envolvimento, sentimento dos filhos e comunicação positiva dos pais aumentam a rejeição clínica. É que os pacientes percebem que o pai é amoroso (sentimento dos filhos), participa da sua vida, dá-lhes apoio, é sensível às suas reações, está presente no seu dia a dia, demonstra seu amor pelo carinho físico e verbalização positiva, como elogios (envolvimento), e se dispõe para diálogo e autonomia do filho (comunicação positiva). Porém, esses pais percebem o transplante como cura total e não conseguem perceber a importância da imunossupressão para manutenção dessa cura. Então, quando o filho não quer tomar a medicação nos horários estipulados diariamente, o pai assume uma atitude apoiadora negligente, o que propicia consequente rejeição clínica.

Nas consultas ambulatoriais é comum o relato das mães e mesmo dos pacientes, sobre a pouca participação do pai em ajudar a seguir o tratamento medicamentoso, fazer os exames e até mesmo comparecer às consultas, assim como observado em uma revisão da literatura brasileira de 64 artigos sobre práticas parentais, apenas em um quarto figurava o pai como participante e somente quatro estudos longitudinais focaram cuidados especiais e doença crônica. ${ }^{37}$

As relações intrafamiliares vêm sofrendo mudanças nas últimas décadas e comportamentos até então compreendidos como culturalmente aceitáveis e esperados, como o uso da força física na educação de crianças por pais e cuidadores, atualmente são 
criticados e coibidos pelos direitos constitucionais, como o Estatuto da Criança e do Adolescente, desde 1990. ${ }^{38}$ No entanto, nesse processo de mudança, a conscientização sobre os danos causados pela punição física é lenta, persistindo a justificação como prática educativa no contexto familiar. ${ }^{38}$ A parentalidade, embora seja um papel exercido no microssistema familiar de Bronfenbrenner, ${ }^{39}$ não deixa de ser uma função social, pois os pais necessitam de informações sobre o desenvolvimento infantil e adolescente e sobre práticas educativas. ${ }^{38}$

As práticas parentais em famílias de crianças com problemas de comportamento são caracterizadas em sua maioria por coerção, disciplina inconsistente, hostilidade, indiferença, negatividade, restrição emocional, pouco afeto e apoio, punição e abuso parental, o que não resolve os problemas de comportamento e leva ainda a mais dificuldades na interação entre genitores e filhos, com menor colaboração, baixa autoestima, baixa autoconfiança e pouca flexibilidade comportamental frente às dificuldades encontradas no cotidiano. ${ }^{40}$

A família pode passar por transições não normativas, circunstâncias estressoras, como insatisfação marital, separação, divórcio, alcoolismo dos pais, doença, dentre outras, que podem exercer um efeito deletério sobre o funcionamento familiar, principalmente pouco monitoramento dos filhos, cuidado parental reduzido e níveis elevados de agressão física. ${ }^{40} \mathrm{O}$ estresse parental é mais elevado nas famílias recasadas e nos padrastos e está fortemente associado a relações de apego inseguras, ao abuso e à parentalidade autoritária e rude, à pobreza, à depressão materna, crianças hiperativas, com doenças crônicas e autismo severo. ${ }^{40}$ Os pais são mais propensos a criticar e a entrar em atrito com seu filho sobre questões menores e sem importância. As relações maritais infelizes aumentam o risco do surgimento de psicopatologias ou problemas de ajustamento. ${ }^{40}$

Exigência, comunicação positiva e punição física praticada pela mãe aumentam a aderência, porém com significância limítrofe $(p=0,06)$. A exigência envolve as regras claras e coerentes que existem na sua família. As duas primeiras variáveis são estratégias educativas com efeitos positivos, enquanto a punição física é uma estratégia educativa inadequada, porém quando associadas com regras claras e coerentes e comunicação positiva contribui com a aderência ao tratamento imunossupressor, porém não deve ser praticada por não ser correta e, além disso, nesta pesquisa ficou demonstrado que contribui para rejeição clínica, pois ao sofrer a punição da mãe, o filho aprende a mentir a fim de evitar apanhar outra vez.

As variáveis do pai de comunicação negativa, responsividade, clima conjugal positivo, punição física e comunicação positiva aumentam a aderência. $\mathrm{O}$ pai usa a comunicação positiva quando permite ao filho um diálogo aberto, permite que se sinta à vontade para falar de si e perguntar o que quiser; é a melhor interação entre pai e filho. ${ }^{38}$ Já a comunicação negativa envolve uma inadequação de conteúdo do diálogo entre pais e filhos pela falta de controle emocional dos pais com ameaças, gritos, xingamentos ou humilhações, que pode culminar ainda com a punição física que não é estratégia educativa ideal, pois é mais um modo dos pais descarregarem suas emoções acumuladas, demonstrando pouca habilidade ou disposição de argumentar e explicar ao filho a situação do conflito que está sendo vivenciada. ${ }^{38} \mathrm{~A}$ responsividade corresponde ao afeto e à participação que o filho percebe receber do pai e o clima conjugal positivo corresponde à boa relação entre o casal com respeito, afeto e diálogo. ${ }^{38}$ Comunicação, responsividade e clima conjugal positivos são estratégias educativas ideais, gerando a espera de um efeito positivo na adesão ao tratamento imunossupressor. Já a comunicação negativa e punição física não são estratégias educativas adequadas, porém quando associadas a estratégias educativas boas tiveram seu efeito minimizado e o resultado final foi adequado. Porém, como já foi citado, a comunicação negativa e a punição física interferem negativamente no desenvolvimento psicológico.

O suporte familiar e as competências de cada membro da família são importantes fontes de informação e influenciam a maneira dos filhos lidarem com a doença hepática e o funcionamento familiar é um previsor importante no ajustamento dos filhos, pois a família pode servir como moderadora na atenuação dos efeitos negativos da doença, promovendo um ambiente facilitador, até mesmo para o seu envolvimento em atividades sociais. ${ }^{41}$

Apenas uma pesquisa mostrou benefícios quando a punição física foi utilizada, que foi a obediência imediata, e 11 comportamentos indesejáveis e experiências associadas a castigos corporais, tais como aumento da agressividade e comportamento delinquente e antissocial, danos na relação entre pais e filhos, a saúde mental prejudicada, aumento do risco de abuso de sua esposa e filhos. ${ }^{42}$ Sob a condição da punição física, as crianças e os adolescentes experimentam menos o amor e sucesso e tendem a ficar geralmente submissas e passivas (embora mudando de comportamento, ocasionalmente, para o oposto extremo de agressão e dominação). ${ }^{43}$ Filhos criados sob tais circunstâncias têm menor probabilidade de serem realistas e efetivos no seu dia-a-dia e têm mais probabilidade de manifestar padrões de comportamento anticonvencionais. ${ }^{43}$ As crianças não nascem preocupadas em ser boas ou más, inteligentes ou estúpidas, amáveis ou não. ${ }^{35}$ Desenvolvem essas ideias e formam autoimagens, baseadas fortemente na forma como são tratadas por pessoas significantes, como os pais, professores e amigos. ${ }^{35}$

Foi observado que o padrão de enfrentamento, a tomada de decisões, as atitudes e o suporte social estão correlacionados à aderência aos níveis séricos do imunossupressor e ao funcionamento psicológico em 152 transplantados hepáticos onde a não aderência foi prevalente ( $73 \%$ à medicação e $47 \%$ aos cuidados de saúde). ${ }^{44}$ Já temperamento de esquiva, a desregulação afetiva e pouco apoio do cuidador são previsores de evolução clínica e mental precária. ${ }^{44}$

O brincar entre adultos e crianças é um meio facilitador para pais e mães educarem seus filhos e representa uma prática educativa em si, já que permite a troca de informações importantes para formação de laços afetivos, socialização e constituição do indivíduo social em seu estudo com famílias de baixa renda em contexto de adversidades. $^{45}$

\section{CONCLUSÃO}

As variáveis do perfil psicológico do paciente e da família interferem na adesão ao tratamento imunossupressor e na rejeição pós-transplante hepático e os pacientes com risco de má aderência ao tratamento imunossupressor e consequente rejeição ao enxerto podem ser beneficiados com atendimentos que busquem melhorar sua saúde mental, a percepção da doença hepática e do transplante e suas atitudes em relação aos seus cuidados de saúde.

AGRADECIMENTOS: Aos professores Dr. Plínio Marco de Toni, Dra Mônica Nunes de Lima e Dra Vânia de Oliveira Gomes de Carvalho. 


\section{ABSTRACT}

The biological, psychological and social developmental changes that occur during the adolescence place the transplanted adolescent at higher risk of non-adherence and poor outcome than other age groups. Purpose: This study tries to assess the importance of agerelated behavioral factors in the patient outcome after liver transplantation. Patients and Methods: Seven instruments were applied to 30 liver transplanted adolescents and young adults (12-30 years old): life satisfaction scale, life orientation scale, resilience scale, self-esteem scale, perceived general self-efficacy scale, exigency and responsiveness scale and quality of familiar interaction scale. Results: In multivariate logistic regression, the variables of life orientation and resilience influence groups with and without clinical rejection $(p=0,03)$. Father's variables that increased adherence were negative communication, responsiveness, positive marital climate, physical punishment and positive communication $(\mathrm{p}=0.03)$, while involvement, feelings of the children and positive communication increased clinical rejection $(\mathrm{p}<0,01)$. For the mother, requirement, positive communication and physical punishment were associated to adherence $(\mathrm{p}=0,06)$. Physical punishment and negative communication increase clinical rejection $(\mathrm{p}=0.02)$. Conclusions: The psychological profile of the patient and family influence adherence to immunosuppression and rejection after liver transplantation and could be used to screen patients at risk for poor adherence to immunosuppression and subsequent graft rejection.

Keywords: Adolescence; Liver Transplantation; Medication Adherence; Adaptation, Psychological; Family Relations; Graft Rejection.

\section{REFERÊNCIAS:}

1. Kamath BM, Olthoff KM. Liver Transplantation in Children: Update 2010 Pediatr Clin N Am. 2010;57:401-14.

2. Miez S. Transplante de fígado. Rev Ass Med Brasil. 1998;44(2):127-34.

3. Saito, MI. Adolescência, cultura, vulnerabilidade e risco: a prevenção em questão. In: SAITO; M. I.; SILVA, L. E. V. (Coords.). Adolescência: Prevenção e risco. São Paulo: Editora Atheneu, 2001:33-38.

4. Casey BJ, Giedd JN, Thomas KM. Structural and functional brain development and its relation to cognitive development. Biol Psychol. 2000;54:241-57.

5. Herculano-Houzel S. O cérebro em transformação. Editora Objetiva 2005.

6. Bell L, Sawyer SM. Transition of care to adult services for pediatric solidorgan transplant recipients. Pediatr Clin N Am. 2010;57:593-610.

7. Knobel M, Aberastury A. La adolescência normal. Argentina, Editorial Paidós, 1970.

8. Hsu, DT. Biological and psychological differences in the child and adolescent transplant recipient. Pediatr Transplantation. 2005;9:416-21.

9. Bell LE, Bartosh SM, Davis CL, Dobbels F, Al-Uzri A, Lotstein D et al. Adolescent Transition to Adult Care in Solid Organ Transplantation: a consensus conference report. Am J Transplant. 2008; 8:2230-42.

10. Dommergues JP, Letierce A, Descott C, Debray D. Suivi medical, vie quotidienne et vecú de jeunes adultes après transplantation hépatique $(\mathrm{TH})$ dans l'enfance. Archives de Pédiatrie. 2008; 15(5):855-6.

11. Diener E, Emmons RA, Larsen RJ, Griffin S. The Satisfaction with Life Scale. Journal of Personality Assessment. 1985; 49:71-5.

12. Scheier MF, Carver CS. Effects of optimism on psychological and physical well-being: Theoretical overview and empirical update. Cognitive Therapy and Research. 1992;16:201-28.

13. Wagnild GM, Young HM. Development and psychometric evaluation of resilience scale. Journal of Nursery Meas, 1999;1:165-78.

14. Pesce RP, Assis SG, Avanci JQ, Santos NC, Malaquias J, Carvalhaes R. Adaptação transcultural, confiabilidade e validade da escala de resiliência. Cadernos de Saúde. 2005;21(2):436-48.

15. Rosenberg M. Society and the adolescent self-image. Princeton, NJ: Princeton University Press, 1965.
16. Rosenberg M. The Rosenberg self-Esteem Scale. Obtained on August 23, 2003 in http://www.bsos.umd.edu/socy/rosenberg.html

17. Nunes R, Schwarzer R, Jerusalem M. 1999. Obtained on March 30, 2005 in http://userpage.fu-berlin.de/ health/auto.htm.

18. Lamborn SD, Mounts NS, Steinberg L, Dornbusch SM. Patterns of competence and adjustment among adolescents from authoritative, authoritarian, indulgent, and neglectful families. Child Development. 1991;62:1049-65.

19. Weber LND, Viezzer AP, Zocche C. Adaptação e validação de duas escalas (Exigência e Responsividade) para avaliar estilos parentais. Em Encontro Brasileiro de Psicoterapia e Medicina Comportamental. Resumos. Campinas: ABPMC. 2002:208.

20. Weber LND, Prado PM, Salvador APV, Branderburg OJ. Construção e confiabilidade das escalas de qualidade na interação familiar. Psicologia Argumento. 2008;26:55-65

21. Weber LND, Salvador APV, Brandenburg O. Qualidade de interação familiar: instrumentos de medida e programas de prevenção. Em M. Bandeira, Z. Del Prette \& A. Del Prette (Orgs.), Estudos sobre habilidades sociais e relacionamento interpessoal. São Paulo: Casa do Psicólogo. 2006:125-214.

22. Dobbels F, Van Damme-Lombaert R, Vanhaecke J, De Geest S. Growing pains: Non-adherence with the immunosuppressive regimen in adolescent transplant recipients. Pediatric Transplantation. 2005;9:381-90.

23. Kaufman M, Shemesh E. The adolescent recipient. Pediatr Clin N Am. 2010;57:575-92.

24. Oliveira VZ, Gomes WB. Comunicação médico-paciente e adesão ao tratamento em adolescentes portadores de doenças orgânicas crônicas. Estud Psicol. 2004;9(3):459-69.

25. Bandeira M, Bekou V, Lott KS, Teixeira MA, Rocha SS. Validação Transcultural do Teste de Orientação da Vida (TOV-R). Estudos de Psicologia 2002;7(2),251-8

26. Patton GC, Tollit MM, Romaniuk H, Spence SH, Sheffield J, Sawyer MG. A prospective study of the effects of optimism on adolescent health risks. Pediatrics. 2011:127(2):308-16.

27. Alonso EM, Neighbors K, Mattson C, Sweet E, Ruch-Ross H, Berry C, Sinacore J. Functional Outcomes of Pediatric Liver Transplantation. J Pediatr Gastroenterol Nutr. 2003;37:155-60. 
28. Lemaneck KL; Kamps J, Chung N.B. Empirically supported treatments in pediatric psychology: regimen adherence. Journal of Pediatric Psychology. 2001;26(5):253-75

29. Comeaux SJ, Jaser SJ. Authonomy and insulin in adolescents with type 1 diabetes. Pediatric Diabetes. 2011;11:498-504.

30. Darling N, Cumsille P, Martinez ML. Individual differences in adolescents' beliefs about the legitimacy of parental authority and their own obligation to obey: a longitudinal investigation. Child Developmental. 2008;79(4):1103-18.

31. Castro EK, Moreno-Jimenez B, Rodriguez-Carvajal R. Psychological well-being in adults transplanted in childhood. Pediatric Transplantation. 2007;11:272-8.

32. Mendes AM, Bousso RS. Not being to live like before: the family dynamics during the experience of pediatric liver transplantation. Ver Latino-Am Enfermagem. 2009;17(1):74-80.

33. Piccinini CA, Castro EK, Alvarenga P, Vargas S, Oliveira VS. A doença crônica orgânica na infância e as práticas educativas maternas. Estudos de Psicologia. 2003;8(1):75-83.

34. Oliveira DS, Rabuske MM, Arpini DM. Práticas de educação: relato de mães usuárias de um serviço público de saúde. Psicologia em Estudo, Maringá. 2007;12(2):351-61

35. Gobitta M, Guzzo RSL. Estudo inicial do inventário de auto-estima (SEI) Forma A. Psicol Reflex Crít. 2002;15(1):143-50.

36. Miranda A, Grau D, Rosel J, Meliá M. Understanding Discipline in Families of Children with Attention-Deficit/Hyperactivity Disorder: A Structural Equation Model. The Spanish Journal of Psychology. 2009;12(2):496-505.
37. Macarini SM, Martins GF, Minetto MFJ, Vieira ML. Práticas parentais: uma revisão da literatura brasileira. Arquivos Brasileiros de Psicologia. 2010;629(1):119-34.

38. Cecconello A, De Antoni C, Koller CL. Práticas educativas, estilos parentais e abuso físico no contexto familiar. Psicologia em Estudo, Maringá. 2003;8:45-54.

39. Bronfenbrenner U. A ecologia do desenvolvimento human: experimentos naturais e planejados. Porto Alegre: Artes Médicas. 1996 (Originally published in 1979.

40. Szelbracikowski AC, Dessen MA. Problemas de comportamento exteriorizado e as relações familiares: revisão de literatura. Psicologia em Estudo, Maringá. 2007;12(1): 33-40.

41. Hoffman RG, Rodrigues JR, Andres JM.; Novak DA. Moderating effects of family functioning on the social adjustment of children with liver disease. Child Health Care. 1995; 24(2):107-117.

42. Gershoff ET. Corporal Punishment by Parents and Associated Child Behaviors and Experiences: A Meta-Analytic and Theoretical Review. Psychological Bulletin. 2002;128(4):539-79.

43. Brandenburg OJ, Weber LND. Revisão de literatura da punição corporal. Interação Psicol. 2005;9(1):91-102.

44. Stilley CS, Dimartini AF, Tarter RE, Devera M, Sereika S, Dew MA, King J, Flynn WB. Liver transplant recipients: individual, social, and environmental resources. Prog Transplant. 2010:20(1):68-74.

45. Martins E, Szymanski I. Brincadeira e práticas educativas familiares: um estudo com famílias de baixa renda. Interações. 2006;11(21):143-64. 